\title{
Particle dynamics in two-dimensional point-loaded granular media composed of circular or pentagonal grains
}

\author{
Ryan Kozlowski ${ }^{1, *}, H u$ Zheng $^{2, * *}$, Karen E. Daniels ${ }^{3, * * *}$, and Joshua E. S. Socolar ${ }^{1, * * * *}$ \\ ${ }^{1}$ Department of Physics, Duke University, Durham, NC 27708 \\ ${ }^{2}$ Department of Geotechnical Engineering, College of Civil Engineering, Tongji University, Shanghai 200092 \\ ${ }^{3}$ Department of Physics, North Carolina State University, Raleigh, NC 27695
}

\begin{abstract}
Granular packings exhibit significant changes in rheological and structural properties when the rotational symmetry of spherical or circular particles is broken. Here, we report on experiments exploring the differences in dynamics of a grain-scale intruder driven through a packing of either disks or pentagons, where the presence of edges and vertices on grains introduces the possibility of rotational constraints at edge-edge contacts. We observe that the intruder's stick-slip dynamics are comparable between the disk packing near the frictional jamming fraction and the pentagonal packing at significantly lower packing fractions. We connect this stark contrast in packing fraction with the average speed and rotation fields of grains during slip events, finding that rotation of pentagons is limited and the flow of pentagonal grains is largely confined in front of the intruder, whereas disks rotate more on average and circulate around the intruder to fill the open channel behind it. Our results indicate that grain-scale rotation constraints significantly modify collective motion of grains on mesoscopic scales and correspondingly enhance resistance to penetration of a local intruder.
\end{abstract}

\section{Introduction}

The response of a granular medium to loading from a single grain highlights fundamental connections across scales from grain-scale dynamics and forces to the macroscopic bulk response of the medium [1]. Simulations and experiments have characterized the dynamics of local intruding rods or grains as a function of parameters including basal friction [2,3], packing fraction [2-4], drive speed [4, 5], drive force $[6,7]$, and driving spring constant for studies of stick-slip dynamics [1-3, 8]. However, in these works and many studies of granular materials in general, the model granular medium is composed of disks or spheres, idealized shapes rarely found in natural granular materials.

Recent studies of granular media composed of grains of a variety of shapes have shown that non-spherical/noncircular grains, which feature additional rotational resistance at the grain-grain interaction scale [9] , better represent measurable bulk parameters like shear strength [1013], relative viscosity [14] in sheared suspensions, and jamming packing fraction [15] observed in real systems.

In this work, we experimentally investigate the role that grain angularity - introducing vertices and flat edges [10] - plays in the dynamical response of a twodimensional (2D) granular medium to a single, spring driven grain. We find that the single-grain intruder is not able to penetrate a packing composed of pentagonal grains

\footnotetext{
*e-mail: rhk11@ @ phy.duke.edu

**e-mail: zhenghu@tongji.edu.cn

***e-mail: kdaniel@ncsu.edu

****e-mail: socolar@duke.edu

A video is available at https://doi.org/10.48448/tn6e-xs20
}

even at low packing fractions relative to a packing composed of disk grains. We investigate the causes of this difference at the grain scale through particle tracking during slip events.

\section{Experimental methods}

\subsection{D granular system with spring driven intruder}

Approximately 1000 bidisperse polyurethane photoelastic disks (or regular pentagons) of thickness $0.6 \mathrm{~cm}$ are set in an annular channel with a width of about 14 small disk diameters, as shown in the schematic diagram in Fig. 1. This is the same apparatus described in Ref. [2]. The channel boundaries are coated in ribbed rubber to prevent particles from slipping. The static friction coefficient between the grains and the glass base on which they rest is $\mu_{\mathrm{BF}}=0.25 \pm 0.05$, and the interparticle friction between grains is $\mu=0.7 \pm 0.1$ for both disks and pentagons. Friction coefficients were obtained by measuring the angle of an inclined surface above which grains slid past other grains that were fixed to the surface. They were measured for edge-edge disk contacts and edge-edge pentagon contacts. $\mu_{\mathrm{BF}}$ was measured for contacts of the flat faces of disk and pentagon grains with a glass base.

A bidisperse mixture of particles is used to prevent crystallization [16]. The number ratio of large to small particles is approximately 1:1.1 for both the packing of disks and the packing of pentagons. The diameters of the small and large disks, respectively, are $d_{\mathrm{Ds}}=1.280 \pm 0.003$ $\mathrm{cm}$ and $d_{\mathrm{Dl}}=1.602 \pm 0.003 \mathrm{~cm}$. The diameters of the 

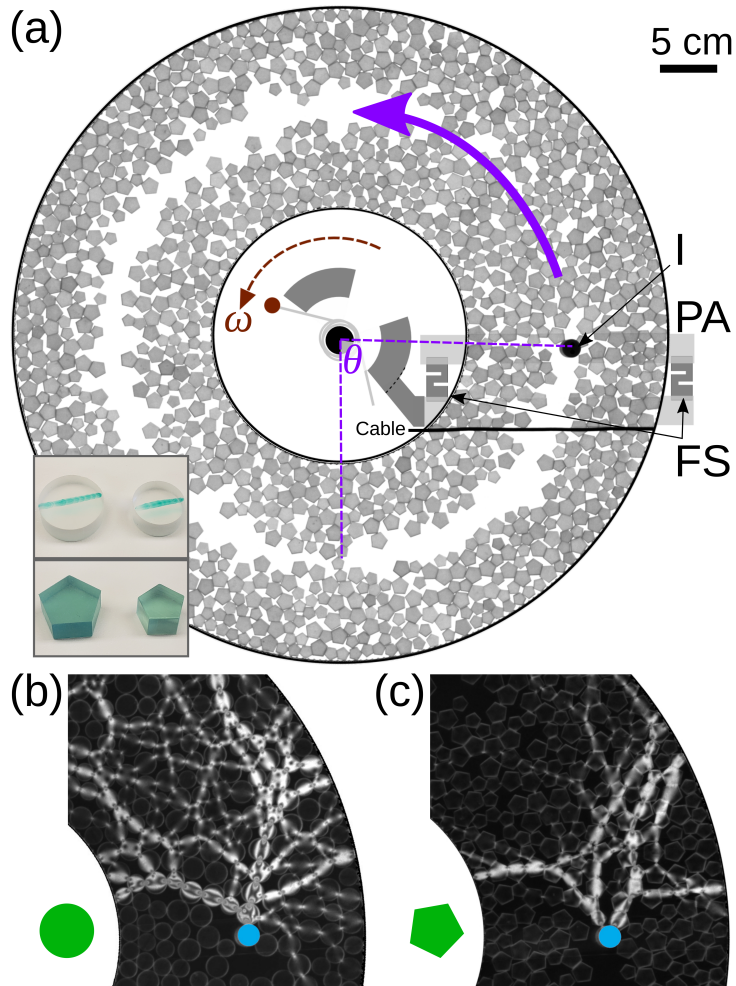

Figure 1. Schematic of apparatus and sample images. (a) The intruder (I) hangs into the granular bed from the pusher arm (PA) and is driven counterclockwise at angular rate $\omega$ by a torque spring. Force sensors (FS) in the pusher arm measure the force of the grains on the intruder. The intruder's angle is $\theta$. The image of the packing of pentagons is a sample image from experiments. Inset: Photographs of disks (top) and pentagons (bottom) used to create bidisperse packings. (b,c) Sample photoelastic images of (b) a packing of disks at $\phi=0.77$ and (c) a packing of pentagons at $\phi=0.65$. The intruder is a blue circle.

circumscribing circles of the small and large regular pentagons, respectively, are $d_{\mathrm{Ps}}=1.211 \pm 0.001 \mathrm{~cm}$ and $d_{\mathrm{Pl}}=$ $1.605 \pm 0.001 \mathrm{~cm}$. The number of grains is varied to change the global packing fraction $\phi$ of the medium, which is defined as the fraction of area in the annular channel that is occupied by grains.

The intruder, a Teflon ${ }^{\circledR}$ rod of diameter $d_{\text {int }}=1.59 \pm$ $0.01 \mathrm{~cm}$, is rigidly fixed to a cantilever that is attached to a post at the center of the annulus, mounted so that it can push the particles without touching the base. The intruder's radial distance from the annulus center is fixed at $R=19.7 \pm 0.1 \mathrm{~cm}$. The cantilever is fitted with s-beam load cells (JINNUO, JLBS-M2) that measure the force of the granular medium on the intruder 100 times per second. A stepper motor (STEPPERONLINE, 24HS343008D) drives one end of a torque spring (stiffness $\kappa_{T}=$ $0.394 \pm 0.001 \mathrm{Nm} / \mathrm{rad}$ ) at a constant angular velocity of $\omega \approx 0.116 \mathrm{rad} / \mathrm{s}$, and we define the drive angular velocity direction, counterclockwise in Fig. 1, to be positive. The other end of the spring pushes the cantilever, driving the intruder through the granular medium. When the medium yields, the elastic energy in the torque spring is released, and the intruder slips rapidly through some distance before
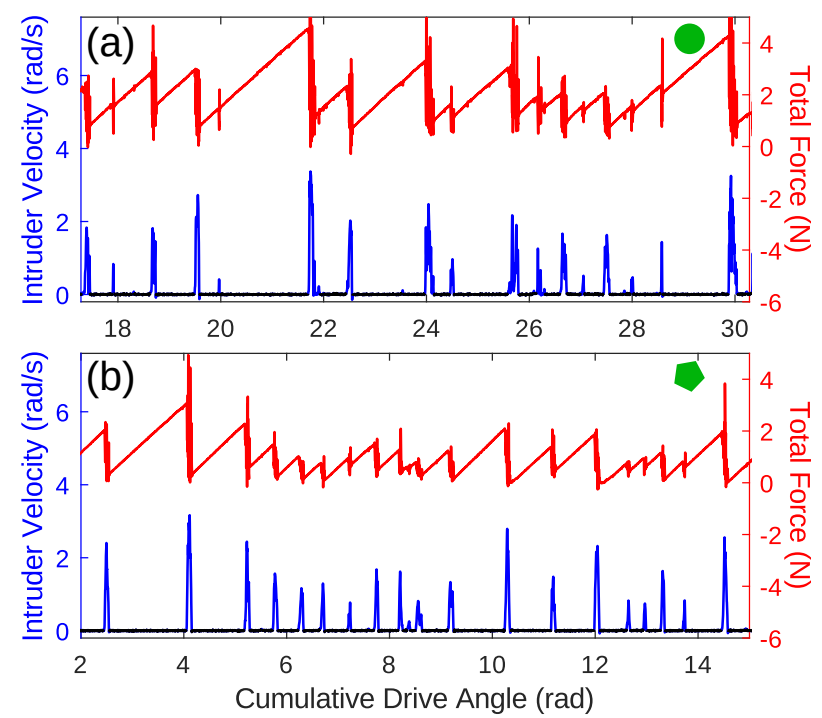

Figure 2. Sample time series measurements of intruder angular velocity (left axis, blue) and force of grains acting on the intruder (right axis, red) for (a) a packing of disks at $\phi=0.77$ and (b) a packing of pentagons at $\phi=0.65$. Black lines at low intruder velocity indicate detected sticking periods.

encountering a jammed configuration of grains that holds it in place as the spring is again loaded.

\subsection{Force data and intruder dynamics}

The granular medium is illuminated from below by a white light LED panel, with the light first passing through a diffuser and circular polarizer. Images are recorded by two cameras above the system. One camera records the light with no further filtering and is used to track the particle and intruder locations, as shown for pentagons in Fig. 1(a). The other camera records photoelastic images of light that passes through a circular polarizer of opposite handedness to the first (the two circular polarizers form a dark-field polariscope [17]), visualizing stresses in each individual grain as demonstrated in Fig. 1(b,c). The angle $\theta$ of the intruder is measured 50 times per second, and the intruder's angular velocity $\dot{\theta}$ is computed by a finite difference of the $\theta(t)$ time series. Sticking periods, in which the intruder is stationary and the spring force increases, are defined by sets of consecutive data points in which $\dot{\theta}$ is below a noise level threshold. Sample time series of intruder velocity and force as measured by the load cells are shown in Fig. 2 as a function of the cumulative drive angle of the stepper motor, which is the cumulative time scaled by $\omega$.

\subsection{Particle tracking}

Individual grains are tracked through template matching via correlation of a post-processed image of the grains with a mask image of the appropriate shape, as demonstrated in Fig. 3. The post-processed image is a bitmap with pixels representing parts of a grain set to +1 and all other pixels set to -1 (Fig. 3(a)). The mask is designed 


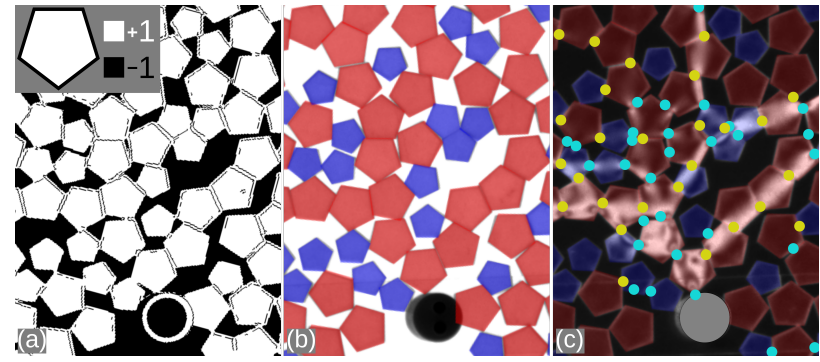

Figure 3. (a) Binarized experimental image. Inset: Pentagon mask at one angle. (b) Original experimental image with transparent detected pentagons; red $=$ large pentagons, blue $=$ small pentagons. (c) Photoelastic image; yellow = edge-edge contacts, cyan $=$ vertex-edge contacts .

with pixels of value +1 representing the template shape as well as an outer shell of pixels set to -1 . Detected particles are iteratively removed from the processed image. Thus, the algorithm finds the highest scoring particles (ones which are not adjacent to many other grains or boundaries because of the -1 shell) first. A large mask is employed first to find the larger grains, and after these particles are located and removed from the processed image a smaller mask is used to find the small grains. If the number of grains is known, the search ends once the proper number has been found; alternatively, if the number of particles varies or imaging conditions are not ideal, one can choose a threshold score for ending the search. The orientations of disks were tracked with an additional processing step after the centers were detected by the above correlation algorithm: the principal moments of inertia of visible bars drawn on each disk were obtained. When applied to the images in this experiment (e.g., Fig. 1(a), which features a cable that partially obscures grains behind the intruder), at least $99 \%$ of particles are detected with position errors on the order of $5 \%$ of the grain size.

\section{Results}

\subsection{Intruder dynamics}

The intruder velocity and force time series in Fig. 2 are plotted at the highest packing fractions attainable for the packings of disks and pentagons, $\phi=0.77$ and $\phi=0.65$ respectively. In either case, higher $\phi$ leads to strong enough jamming that the packing buckles and particles pop out of the monolayer. Although these are significantly different packing fractions, the intruder exhibits qualitatively similar stick-slip dynamics, characterized by sequences of sticking periods in which the intruder is nominally stationary and slip events in which the intruder slips through the medium, for the two different particle shapes. (For disks, however, a packing fraction near $\phi=0.65$ yields intermittent flow, in which the intruder moves through the system with only occasional clogging events [2,3].)

As demonstrated in Fig. 1, the intruder leaves a channel in its wake as it pushes through the system; local packing fractions outside of the channel and for a stable cluster
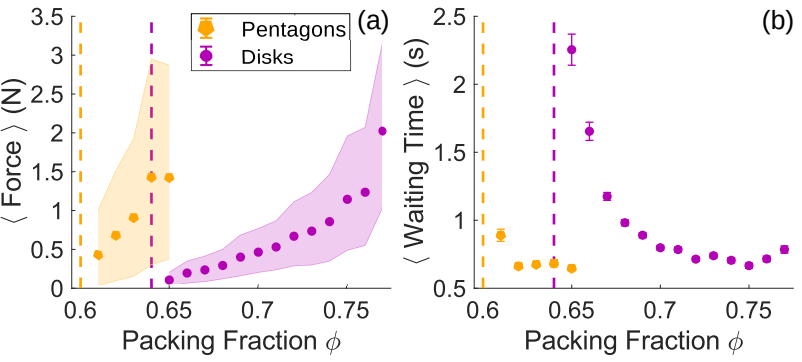

Figure 4. (a) Average force on the intruder for disks (purple filled circles) and pentagons (orange filled pentagons). The range of data is from the lower 10\% cutoff to the upper $90 \%$ cutoff of the intruder's force distribution. (b) Average waiting time of the intruder between consecutive sticking periods. Error bars represent standard errors of the average. Dashed vertical lines indicate packing fractions at which a completely free channel forms within a given experimental run.

of grains in front of the intruder are significantly closer to the expected frictional jamming fraction $\sim 0.78$ [15]. The difference between the global packing fractions for disks and pentagons can thus be largely attributed to the fact that the size of the cluster of particles in front of the intruder is significantly smaller for pentagons than for disks.

Figure 4 shows the average force on the intruder and the average waiting time between sticking periods for each shape at varying $\phi$. The forces and fluctuations of forces for the packing of pentagons are comparable to those with disks at significantly higher packing fractions. Moreover, for the packing of pentagons, the transition from stick-slip dynamics to free channel formation occurs over a very small range of $\phi$. Unlike in the case of disks, no regime in which there are long waiting times between clogging events is observed; the average waiting time never exceeds $1 \mathrm{~s}$, the typical duration of a slip event. We note, however, that preliminary simulations performed by collaborators suggest that intermittent clogging dynamics would occur for pentagons in wider channels [18].

\subsection{Particle flow fields}

In order to understand why pentagons exhibit strong stickslip dynamics even with a small cluster of grains in front of the intruder, we search for clues in the dynamics of individual grains. Average fields of speed, rotational speed of grains about their centers, and vorticity were produced from over 400 slip events, shown in Fig. 5 for the packing of disks at $\phi=0.77$ and the packing of pentagons at $\phi=0.65$. For each slip event, a single representative frame was chosen to contribute to the overall average among slip events; the exact choice of which frames to include does not influence the presented results. Every image is rotated so that the intruder is at the same angle prior to averaging.

The most striking difference between the flow fields for packings of disks and packings of pentagons during slip events is that pentagons primarily yield by moving forward, as indicated by the dark band in Fig. 5(d) extending to $\pi / 2$ radians in front of the intruder, while disks are 
(a)

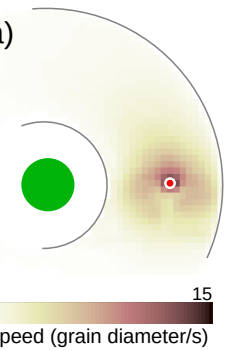

(d)

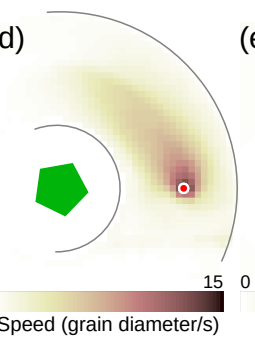

(b)

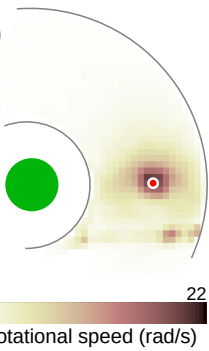

(e)

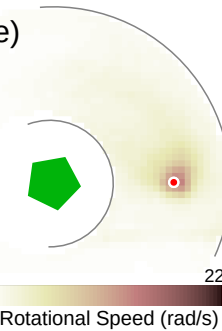

(c)

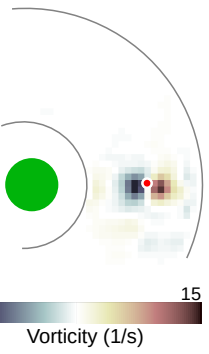

(f)

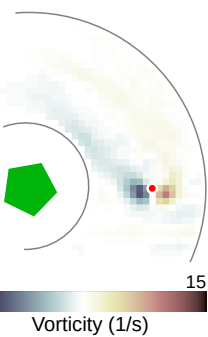

Figure 5. (a,b,c) Average flow (speed) field, average rotational speed field, and average vorticity field for flow of disk grains at $\phi=0.77$ during slip events around the intruder (red circle). (d,e,f) Same, for pentagonal grains at $\phi=0.65$.

able to circulate around the intruder without extensive forward motion. The higher magnitude of rotational speed in the proximity of the intruder indicates that disks rotate more than pentagons. Lastly, the vorticity from the average flow field shows a stronger magnitude of circulation for disks than for pentagons. All of these results suggest that the pentagons are unable to circulate around the local load due to local rotational constraints, leading to a greater yield strength even with a significantly smaller cluster of grains in front of the intruder.

\section{Conclusion and outlook}

We have shown that pentagonal grains resist the penetration of a grain-scale intruding rod at significantly lower global packing fractions than do disks. This is evidenced by large forces and small waiting times between sticking periods at $\phi \sim 0.65$ for pentagons, with comparable dynamics at $\phi \sim 0.77$ for disks. By applying a correlation template matching method to experimental images of grains, we have linked the observed intruder dynamics to grain displacements and rotations during slip events. Compared with disks, rotations of pentagonal grains are smaller and pentagons are less likely to circulate around the intruder to fill the open channel behind it. Hence, pentagons collectively yield by being pushed forward into the channel that wraps nearly around the annular channel. This provides an explanation for why a smaller cluster of grains in front of the intruder (and therefore $\phi$ ) compared with disks, in which the annulus is nearly completely filled, still hinders intruder penetration.

Future work will explore the correlations between particle motion and the emergent system-spanning force networks responsible for observed stick-slip dynamics, obtained from photoelastic imaging (Fig.1(b,c)) and grain tracking (Fig.3(c)). The sample images in Fig. 1(b,c) demonstrate qualitative differences between the force networks of jammed (stuck) packings of disks or pentagons that complement the slip event flow field differences of Fig. 5; quantification of these differences is underway.

More broadly, it would be interesting to explore the dependence of granular intrusion dynamics on other aspects of the packing material, such as grain anisotropy (which constrains relative rotations on the grain scale differently from grain angularity) and packing dispersity, and on the system size.

\section{Acknowledgements}

This work was supported by the US Army Research Office through grant W911NF1810184.

\section{References}

[1] A. Tordesillas, J.E. Hilton, S.T. Tobin, Phys. Rev. E 89, 042207 (2014)

[2] R. Kozlowski, C.M. Carlevaro, K.E. Daniels, L. Kondic, L.A. Pugnaloni, J.E.S. Socolar, H. Zheng, R.P. Behringer, Phys. Rev. E 100, 032905 (2019)

[3] C.M. Carlevaro, R. Kozlowski, L.A. Pugnaloni, H. Zheng, J.E.S. Socolar, L. Kondic, Phys. Rev. E 101, 012909 (2020)

[4] E. Kolb, P. Cixous, N. Gaudouen, T. Darnige, Phys. Rev. E 87, 032207 (2013)

[5] A. Seguin, C. Coulais, F. Martinez, Y. Bertho, P. Gondret, Phys. Rev. E 93, 012904 (2016)

[6] R. Candelier, O. Dauchot, Phys. Rev. E 81, 011304 (2010)

[7] C.J. Olson Reichhardt, C. Reichhardt, Phys. Rev. E 82, 051306 (2010)

[8] I. Albert, P. Tegzes, R. Albert, J.G. Sample, A.L. Barabási, T. Vicsek, B. Kahng, P. Schiffer, Phys. Rev. E 64, 031307 (2001)

[9] N. Estrada, E. Azéma, F. Radjai, A. Taboada, Phys. Rev. E 84, 011306 (2011)

[10] T. Binaree, E. Azéma, N. Estrada, M. Renouf, I. Preechawuttipong, Phys. Rev. E 102, 022901 (2020)

[11] K.A. Murphy, K.A. Dahmen, H.M. Jaeger, Phys. Rev. X 9, 011014 (2019)

[12] K. Mair, K.M. Frye, C. Marone, J. Geophys. Res. Solid Earth 107, ECV 4 (2002)

[13] C. Wang, K. Dong, A. Yu, Phys. Rev. E 92, 062203 (2015)

[14] A. Singh, C. Ness, R. Seto, J.J. de Pablo, H.M. Jaeger, Phys. Rev. Lett. 124, 248005 (2020)

[15] Y. Zhao, J. Barés, H. Zheng, C.S. Bester, Y. Xu, J.E.S. Socolar, R.P. Behringer, Gran. Matt. 21 (2019)

[16] C.S. O’Hern, L.E. Silbert, A.J. Liu, S.R. Nagel, Phys. Rev. E 68, 011306 (2003)

[17] K.E. Daniels, J.E. Kollmer, J.G. Puckett, Rev. Sci. Inst. 88, 051808 (2017)

[18] M. Carlevaro, L. Pugnaloni, private communication. 